\title{
TARGETING KEY AROMATIC SUBSTANCES ON THE TYPICAL AROMA OF SHERRY VINEGAR
}

\author{
RAQUEL M. CALLEJÓN ${ }^{1}$, M. Lourdes Morales ${ }^{1}$, Ana M. Troncoso ${ }^{1}$, A. C. Silva Ferreira ${ }^{2 *}$ \\ ${ }^{1}$ Área de Nutrición y Bromatología. Facultad de Farmacia. Universidad de Sevilla. c/ P. \\ García González n² , E- 41012. Sevilla. Spain. ${ }^{2}$ Escola Superior de Biotecnología, \\ Universidade Católica Portuguesa. R. Dr. Antonio Bernardino de Almeida, 4200-072 Porto, \\ Portugal.
}

(*) Corresponding author: raqcalfer@alum.us.es

Keywords: Aroma; Sherry wine vinegar; GC-O; Sotolon

Targeting substances with large impact on the perceived quality of a food product constitutes one of the most challenging tasks in flavor research. The main difficulty is found on the fusion between sensory and chemical data. In this work two gas chromatographyolfactometry (GC-O) techniques were used to screen substances with high impact on the perceived quality of Sherry vinegar: aroma extract dilution analysis (AEDA) and frequency counting (FC) using simultaneously detection using two exits on a customized "multipost" Sniffer ODO-II.

The GC-O study revealed the presence of 108 aromatic notes among which 64 were possible to describe by the panelists. Good correlations were observed among the simultaneous AEDA $(r>0.90)$ the same observation is not extensive to the FC with AEDA ( $r$ $<0.45$ ). This can be to a certain extent attributed to the presence of ambiguous descriptors that presented a low FC but high dilution factors. Diacetyl, isoamyl acetate, acetic acid and sotolon, reached the highest frequency and FD factors. On other hand ethyl acetate displays the highest frequency (9/9) but only a FD factor of 4 . To test the sensory impact of these odorants, they were added to a $7 \%(\mathrm{v} / \mathrm{v})$ acetic acid solution. Similarity value (SV) between solutions and the Sherry vinegar was determined. The highest value from the similarity test was observed when diacetyl, ethyl acetate and sotolon was added simultaneously. The profile of this model solution and the Sherry vinegar showed a good similarity in the "tipicity" descriptor, which emphasizes the important contribution of these 3 compounds to the global aroma of this vinegar.

\section{INTRODUZIR REFS}

References: (Times New Roman 11 pt)

Example:

(1) Amrein TM, Limacher A, Conde-Petit B, Amadò R, Escher F (2006). Influence of thermal processing conditions on acrylamide generation and browning in a potato model system. J. Agric.

Food Chem. 54, 5910-5916. 